\title{
Synthesis and Characterization of Fluoridated Silver Nanoparticles and Their Potential as a Non-Staining Anti-Caries Agent
}

This article was published in the following Dove Press journal: International Journal of Nanomedicine

\author{
Iris Xiaoxue Yin $\mathbb{D}^{1,2}$ \\ Irene Shuping Zhao $\mathbb{I D}^{3}$ \\ May Lei Mei (D) ${ }^{4}$ \\ Edward Chin Man Lo $\mathbb{D}^{\prime}$ \\ Jinyao Tang $\mathbb{D}^{5}$ \\ Quanli $\mathrm{Li}^{6}$ \\ Lok Yan So ${ }^{7}$ \\ Chun Hung Chu $\mathbb{D}^{1,2}$ \\ 'Faculty of Dentistry, The University of \\ Hong Kong, Hong Kong, People's \\ Republic of China; ${ }^{2} \mathrm{HKU}$ Shenzhen \\ Institute of Research and Innovation, \\ Shenzhen, People's Republic of China; \\ ${ }^{3}$ School of Dentistry, Shenzhen \\ University Health Science Center, \\ Shenzhen, People's Republic of China; \\ ${ }^{4}$ Faculty of Dentistry, University of \\ Otago, Dunedin, New Zealand; \\ ${ }^{5}$ Department of Chemistry, The \\ University of Hong Kong, Hong Kong, \\ People's Republic of China; ${ }^{6}$ School of \\ Stomatology, Anhui Medical University, \\ Hefei, People's Republic of China; \\ ${ }^{7}$ Department of Applied Biology and \\ Chemical Technology, The Hong Kong \\ Polytechnic University, Hong Kong, \\ People's Republic of China
}

Correspondence: May Lei Me

Department of Oral Rehabilitation, Faculty of Dentistry, University of Otago,

Dunedin, New Zealand

Tel +6434794196

Fax +6434795079

Email may.mei@otago.ac.nz

Chun Hung Chu

Faculty of Dentistry, The University of Hong Kong, 34 Hospital Road, Hong Kong SAR, People's Republic of China

Tel +852 28590287

Fax +852 28582532

Email chchu@hku.hk
Objectives: The first objective of this study was to prepare sodium fluoride (NaF) solution with various concentrations of polyethylene glycol-coated silver nanoparticles (PEG-AgNPs). The second objective was to study the antibacterial activity against Streptococcus mutans and the tooth-staining effect of the solution.

Methods: PEG-AgNPs were prepared via the one-step chemical reduction of silver acetate with thiolated polyethylene glycol. The PEG-AgNPs were characterized with ultravioletvisible spectrometry and transmission electron microscopy. The half maximal inhibitory concentration (IC50) for the PEG-AgNPs against Streptococcus mutans and human gingival fibroblasts (HGF-1) were determined. The staining effect on dentin and enamel for the $2.5 \%$ $\mathrm{NaF}$ solutions with PEG-AgNPs at 12,800, 6400, 1600, and $400 \mathrm{ppm}$ was investigated using digital spectrophotometry. The IC50 of the fluoridated silver nanoparticles against Streptococcus mutans were measured.

Results: The PEG-AgNPs have an average diameter of $2.56 \pm 0.43 \mathrm{~nm}$ and showed excellent stability at high ionic strength $(2.5 \% \mathrm{NaF})$ for 18 months. The IC50 of PEG-AgNPs against Streptococcus mutans was found to be $21.16 \pm 1.08 \mathrm{ppm}$ silver, which was half of IC50 against HGF-1 cells $(42.36 \pm 1.12 \mathrm{ppm})$, providing a working range to kill bacteria with no harm to human cells. The formulations with different concentrations of PEG-AgNPs showed no significant staining of teeth. Combining PEG-AgNPs with NaF significantly expanded the therapeutic window against Streptococcus mutans by reducing its IC50.

Conclusion: A biocompatible solution of NaF with PEG-AgNPs was developed. Because it has antibacterial activity against Streptococcus mutans and no tooth-staining effect, it can be used as an anti-caries agent.

Keywords: silver, nanoparticles, fluoride, dentin, caries, antibacterial

\section{Introduction}

The antibacterial property of silver metal, ions, or silver-containing compounds has historically been well recognized, but the mechanism of their action is not fully understood. It has been proposed that ionic silver causes a structural change in the bacterial cell membrane; enters the cell and forms electron-dense granules around the cell wall and cytoplasm; stops deoxyribonucleic acid replication by condensing it; attacks thiol groups in enzymes, which leads to the inactivation of corresponding enzymatic activity; and eventually causes cell death. The antimicrobial effect of silver metal and silver-containing compounds relies on the release of bioactive silver ions. ${ }^{1}$ Antibacterial silver has been employed together with fluorides in 
dentistry for the prevention and arrest of caries. It has been suggested that silver ions mainly target cariogenic bacteria, whereas fluoride ions help with rebuilding the tooth structure. ${ }^{2}$ Silver diamine fluoride (SDF) has been used to arrest caries due to the advantages of effectiveness, low cost, noninvasiveness, and ease of application. ${ }^{3}$ The black staining of a caries lesion on a tooth due to the deposition of silver chloride, however, has significantly limited the wide clinical application of SDF. ${ }^{4}$ This can be avoided by using the nano-sized silver particles and protecting them using a capping agent.

A nanoparticle whose diameter ranges from 1 to $100 \mathrm{~nm}$ can provide novel properties owing to its small size and enormous surface area. ${ }^{5}$ These nanoparticles can be made into various shapes, including a sphere, rod, triangle, and wire, and various capping agents, such as a citrate, polymer, and protein, can be used to protect the nanoparticles' surface depending on the desired chemical and biological properties. ${ }^{6}$ Silver nanoparticles (AgNPs) have been used as some of the most common nanoparticles in today's commercial products, especially in the medical field, for wound dressings, drug delivery, imaging, and biosensing. ${ }^{7}$ AgNPs also draw attention from dental researchers due to their potential utilization for anti-caries. AgNPs can inhibit the growth and adhesion of cariogenic bacteria, especially Streptococcus mutans (S. mutans). ${ }^{8}$ A number of studies have investigated the addition of silver nanoparticles into adhesives, toothpaste, and restorative materials to prevent caries. ${ }^{9}$ Researchers have recently explored the cooperation of AgNPs and fluoride to arrest caries, and this included the combination of an antibacterial and remineralizing agent. ${ }^{10}$

It is known that the uncoated or electrostatically stabilized (e.g., with citrate) AgNPs aggregate under high ionic strength conditions. To be used with fluorides, AgNPs must be stabilized with an appropriate capping ligand that possesses a covalent binding group and long polymeric chains. Polyethylene glycol (PEG) has been reported to increase the stability of AgNPs such that they could be preserved even at high ionic concentrations. ${ }^{5}$ PEG is a safe, water-soluble, and biocompatible polymer that is approved by the Food and Drug Administration (FDA) of the United States for use in food and pharmaceutical products. $^{11}$ PEG-coated AgNPs (PEG-AgNPs) were shown to be less toxic than those coated with other capping agents and silver ions. ${ }^{12}$ Therefore, PEG is a suitable capping agent for AgNPs. Moreover, a silver core protected by PEG would be less liable to oxidize; thus, less coloring side reactions are expected. This aim of this study is to create a novel anti-caries agent using PEG-AgNPs and fluoride with no tooth-staining effect.

\section{Materials and Methods Synthesis of Fluoride Solution with Silver Nanoparticles}

The synthesis of PEG-AgNPs was carried out via the one-step chemical reduction of silver acetate with polyethylene glycol thiol (PEG-SH, average molecular weight 800). The PEG-SH was used as a reducing agent and as a coating agent for the stabilization of AgNPs. To prepare PEG-AgNPs, 20-mg PEG$\mathrm{SH}$ and 40-mg silver acetate were dissolved individually in 400-mL ethanol. The silver acetate solution was kept in darkness to avoid decomposition and was stirred at $4{ }^{\circ} \mathrm{C}$ until the homogeneous phase resulted. After mixing the two solutions, the color changed immediately from colorless to light yellow, indicating the formation of AgNPs. The reaction mixture was stirred for 18 hours at $4{ }^{\circ} \mathrm{C}$ under ambient light to ensure the formation and even the growth of nanoparticles. The prepared PEG-AgNPs were concentrated via rotary evaporation to a final volume of $c a .50 \mathrm{~mL}$. The ethanolic solvent was exchanged to water, followed by an additional concentration of PEG-AgNPs 10-fold using an Amicon ultra centrifugal filter of MWCO $30 \mathrm{kDa}$. The unreacted starting reagents were also removed at this stage via washing three times with distilled water. To this PEG-AgNPs solution, sodium fluoride ( $\mathrm{NaF} 2.5 \%$ ) was added to a final concentration of 11,310 ppm fluoride to yield PEG-AgNPs solution of various silver concentrations $(12,800,6400,1600$, and $400 \mathrm{ppm})$.

\section{Characterization of the Silver Nanoparticles}

The ultraviolet-visible (UV-vis) spectrometry characteristics of PEG-AgNPs were evaluated with an Agilent Cary 4000 UV-vis spectrophotometer. Transmission electron microscopy (TEM) images and energy-dispersive x-ray spectroscopy (EDS) were recorded using a JEOL JEM 2100F (field emission) scanning transmission electron microscope (STEM) equipped with an Oxford INCA x-sight EDS Si(Li) detector at an acceleration voltage of $200 \mathrm{kV}$ to evaluate the morphology and size of PEG-AgNPs. A diluted PEG-AgNPs sample in water with a total of $10 \mu \mathrm{L}$ was deposited onto TEM copper grids with a lacy carbon film. The sample was allowed to dry overnight in darkness before being analyzed using STEM. The average size of PEG-AgNPs was obtained by measuring the diameters in a representative TEM image with Image $\mathrm{J}$ (software from the National Institute of Health). The stability 
of PEG-AgNPs was measured using TEM images taken at 18 months after synthesis. The silver concentration in PEGAgNPs samples was determined by digesting the sample in $1 \%$ nitric acid and analyzing it using inductively coupled plasma optical emission spectroscopy.

\section{Antibacterial Test}

S. mutans (ATCC 35668) was cultured in brain heart infusion media and agar. ${ }^{13}$ Half maximal inhibitory concentration (IC50) of PEG-AgNPs and fluoridated PEG-AgNPs against $S$. mutans, which are defined as the concentration of a drug required for $50 \%$ inhibition in vitro, were determined via cell turbidity measured at OD600 nm. SDF was used as a positive control. The IC50s of silver nitrate $\left(\mathrm{AgNO}_{3}\right)$ and $\mathrm{NaF}$ were also determined for comparison. The experiments were performed in 96-well plates with the serial dilution of testing samples across the columns. Each dilution was performed in duplicate. In each well, $89 \mu \mathrm{L}$ of medium were added together with $10 \mu \mathrm{L}$ of the starting culture (to yield a final CFU/mL of ca. $5 \times 10^{6}$ ) and $1 \mu \mathrm{L}$ of the testing sample. Controls with the bacterium and medium only were included to ensure the viability of the bacterium and the sterility of the medium. The plate was then incubated at $37^{\circ} \mathrm{C}$ for 18 hours in darkness. The absorbance at $600 \mathrm{~nm}$ was measured using a Bio-Rad Model 680 microplate reader before and after the incubation. The experiment was repeated three times to ensure consistency.

\section{Cell Cytotoxicity Test}

Human gingival fibroblasts (HGF-1) (ATCC ${ }^{\circledR}$ CRL-2014 ${ }^{\mathrm{TM}}$ ) were maintained in Dulbecco's modified eagle medium (DMEM) containing 10\% fetal bovine serum (FBS), $100 \mathrm{U} /$ $\mathrm{mL}$ penicillin, and $100 \mu \mathrm{g} / \mathrm{mL}$ streptomycin. The cell lines were cultured at $37{ }^{\circ} \mathrm{C}$ in a humidified atmosphere with $5 \%$ carbon dioxide. The 3-(4,5-dimethylthiazole-2-yl)-2,5-diphenyltetrazoli-umbromide (MTT) assay was used to investigate the cytotoxic effect of PEG-AgNPs. Briefly, 5000 HGF-1 cells were seeded in a 96-well plate and cultured in DMEM containing 10\% FBS for 24 hours for cell attachment. Cells were then treated with various concentrations of PEG-AgNPs (2.5, 5, 10, 20, 40, 80, and $160 \mathrm{ppm} \mathrm{Ag}), \operatorname{NaF}$ (0.0156, 0.0313, $0.0625,0.125$, and $0.25 \% \mathrm{w} / \mathrm{v})$, SDF $(0.000475,0.00095$, $0.0019,0.0038$, and $0.0076 \% \mathrm{w} / \mathrm{v})$, and a culture medium as the control for 5 minutes. The sample medium was then removed, and cells were washed once with phosphate buffered saline (PBS) and replaced with a culture medium for another 24 hours. To measure the cytotoxicity, $0.5 \mathrm{mg} / \mathrm{mL}$ MTT were added to each well and incubated for 4 hours at $37^{\circ} \mathrm{C}$. The resulting crystal formazan was then solubilized in $100 \mu \mathrm{L} 10 \%$ sodium dodecyl sulfate containing $0.01 \mathrm{M}$ hydrochloric acid. Absorbance was measured at $570 \mathrm{~nm}$ with $655 \mathrm{~nm}$ as the reference wavelength using a Bio-Rad Model 680 microplate reader. The relative viability of cells was presented as follows:

$$
\frac{\text { Average optical density of test group }}{\text { Average optical density of control group }} \times 100 \%
$$

\section{Coloring Test}

Extracted sound human third molars were collected with the patients' written informed consent. Teeth were stored in distilled water at $4{ }^{\circ} \mathrm{C}$ until use and were used within one month after extraction. Eighteen slices of enamel and dentin with a thicknesses of $2 \mathrm{~mm}$ were prepared and polished. The polished slices were examined using a stereomicroscope to exclude slices with cracks or other defects. Testing compounds with a total of $20 \mu \mathrm{L}$ were added to each slice and were allowed to dry for 1 hour at $25{ }^{\circ} \mathrm{C}$ under ambient light. Four testing solutions, which were 12,800 ppm AgNPs + 2.5\% NaF, 6400 ppm AgNPs $+2.5 \%$ NaF, 1600 ppm AgNPs $+2.5 \% \mathrm{NaF}$, and $400 \mathrm{ppm}$ AgNPs $+2.5 \% \mathrm{NaF}$, were prepared and examined. $38 \%$ SDF was used as the positive control, and water was used as the negative control. The color of treated slices was examined with the VITA Easyshade advance portable dental spectrophotometer (VITA Zahnfabrik GmbH, Bad Säckingen, Germany). The color of each tooth was elucidated three-dimensionally in space, according to the Commission International del'Eclairage $L^{*} a^{*} b^{*}$ color system. The $L^{*}$ axis represented lightness ranging from black (0) to white (100), the a* axis described red $\left(+a^{*}\right)$ to green $\left(-a^{*}\right)$, and the $b^{*}$ axis represented yellow $\left(+b^{*}\right)$ to blue $\left(-b^{*}\right)$. The instrument was calibrated according to the manufacturer's instruction before application. The $\mathrm{L}^{*}, \mathrm{a}^{*}, \mathrm{~b}^{*}$, hue $\left(\mathrm{h}^{*}\right)$ and chroma $\left(\mathrm{C}^{*}\right)$ values were measured for triplicate samples, and average values were recorded. The difference in color between testing groups and the negative control was calculated based on the mathematical equation: $^{14}$

$$
\Delta E^{*}=\sqrt{\left(\Delta L^{*}\right)^{2}+\left(\Delta a^{*}\right)^{2}+\left(\Delta b^{*}\right)^{2}}
$$

\section{Statistical Analysis}

Shapiro-Wilk was used to assess the normal distribution for all data $(\mathrm{p}>0.05)$. Pairwise $t$-test was employed to assess the $\mathrm{L}^{*} \mathrm{a}$ *and $\mathrm{b}^{*}$ values between $\mathrm{T} 0$ and $\mathrm{T} 1$ within each group. One-way ANOVA with Bonferroni multiple 
comparison tests was used to compare the values of MIC, MBC, IC50, and color change among different groups. All data were analyzed with IBM SPSS V20.0 software (IBM Corporation, Armonk, NY, USA), and the level of significance was set at $5 \%$.

\section{Results}

\section{Characterization of Fluoridated PEG-AgNPs}

After mixing the silver acetate and PEG-SH ethanolic solutions, a pale yellow color was immediately observed, indicating the formation of PEG-AgNPs. The UV-vis absorption spectrum of the synthesized PEG-AgNPs in water (Figure 1) did not show any characteristic surface plasmon resonance (SPR) band.

Figure $2 \mathrm{~A}$ and $\mathrm{B}$ shows the representative TEM images of PEG-AgNPs in water, and Figure $2 \mathrm{C}$ shows the size distribution histogram of corresponding particles. The TEM analysis clearly revealed the formation of monodispersed spherical PEG-AgNPs with a diameter of $2.56 \pm 0.43 \mathrm{~nm}$. The presence of PEG-SH on the surface of AgNPs was confirmed via elemental analysis using EDS during TEM. It is noteworthy that there was no detectable aggregation of PEG-AgNPs. The

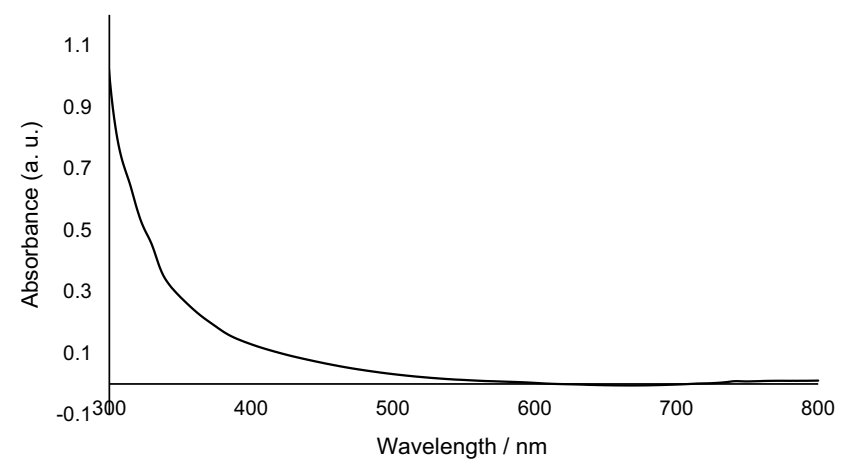

Figure I UV-vis spectrum of PEG-AgNPs.

silver content of PEG-AgNPs reached up to $14,000 \mathrm{ppm}$ after the concentration using a centrifugal filter.

\section{Stability of PEG-AgNPs with $\mathrm{NaF}$}

The incorporation of NaF into the PEG-AgNPs did not cause any precipitation of nanoparticles. The compatibility of PEG-AgNPs with $2.5 \% \mathrm{NaF}$ was confirmed with the TEM images shown in Figure 2D-F, where no aggregation and no significant change in the sizes of AgNPs were found after the addition of $\mathrm{NaF}(2.56 \pm 0.43$ and $2.60 \pm 0.51 \mathrm{~nm}$ without and with the addition of NaF, $p=0.54$ ). The PEG-AgNPs also showed high stability in $2.5 \% \mathrm{NaF}$ stored in darkness at

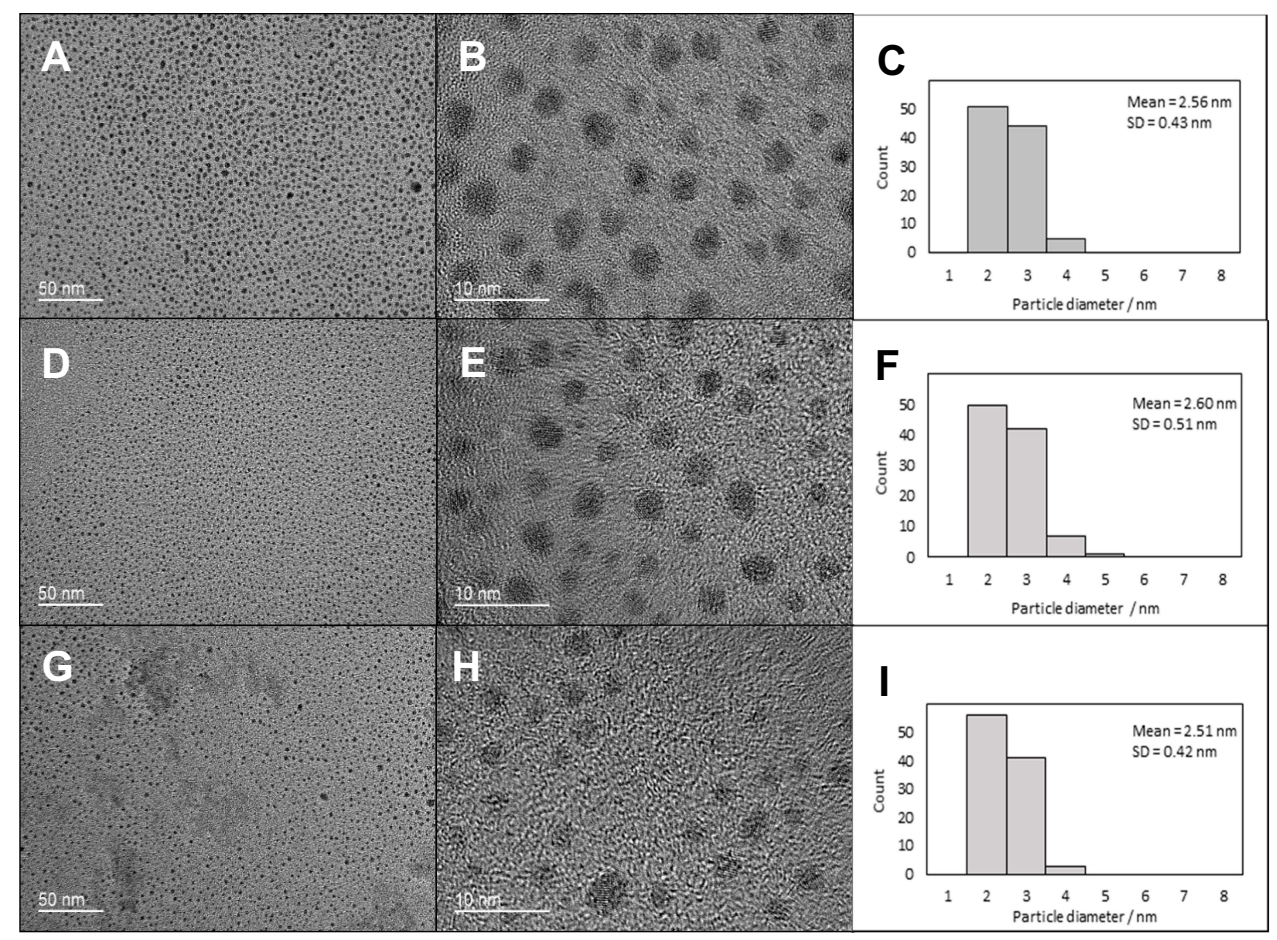

Figure 2 Typical TEM images of PEG-AgNPs and corresponding size distribution. (A, B) TEM images of freshly prepared PEG-AgNPs in water; (C) size distribution of freshly prepared PEG-AgNPs in water; (D, E) TEM images of freshly prepared PEG-AgNPs in $2.5 \%$ NaF; (F) size distribution of freshly prepared PEG-AgNPs in $2.5 \%$ NaF; (G, H) TEM images of PEG-AgNPs in $2.5 \% \mathrm{NaF}$ for 18 months; (I) size distribution of PEG-AgNPs in $2.5 \% \mathrm{NaF}$ for 18 months). 
$4{ }^{\circ} \mathrm{C}$ for 18 months (Figure $2 \mathrm{G}-\mathrm{I}$ )). The size of fluoridated PEG-AgNPs $(2.51 \pm 0.42 \mathrm{~nm})$ has not demonstrated statistically significant difference compared to freshly prepared PEG-AgNPs with NaF $(p=0.17)$.

\section{Antibacterial and Cytotoxic Effect}

The antibacterial and cytotoxic effects of PEG-AgNPs against $S$. mutans and HGF-1 were determined, and the corresponding values of IC50 are listed in Table 1. PEGAgNPs showed the inhibition against the growth of $S$. mutans, and the IC50 was found to be $21.16 \pm 1.08$ ppm Ag, which was about half of that against HGF-1 $(42.36 \pm 1.12 \mathrm{ppm} \mathrm{Ag}, p<0.001)$. SDF exhibited a lower IC50 value of $9.67 \pm 1.55 \mathrm{ppm} \mathrm{Ag}$ against S. mutans, but it also had a lower IC50 value against HGF-1 (32.78 ppm Ag, $p<0.001$ ). It is noteworthy that $\mathrm{AgNO}_{3}$ is highly toxic toward HGF-1. The IC50 of $\mathrm{AgNO}_{3}$ against $S$. mutans was $7.13 \pm 2.16 \mathrm{ppm} \mathrm{Ag}$, whereas that against HGF-1 was $0.23 \mathrm{ppm} \mathrm{Ag}(p<$ 0.001 ). NaF was able to inhibit $50 \%$ of the growth of $S$. mutans at $32.96 \pm 2.86 \mathrm{ppm} \mathrm{F}$ and did not damage $50 \%$ of the HGF-1 at 1131 ppm F.

\section{Combinational Effect of PEG-AgNPs and $\mathrm{NaF}$}

The antibacterial effects of four fluoridated PEG-AgNPs solutions containing various ratios of PEG-AgNPs $(12,800$, 6400,1600 , and $400 \mathrm{ppm} \mathrm{Ag}$ ) and $\mathrm{NaF}$ (fixed at $2.5 \%=$
11,310 ppm F) were determined, and the corresponding values of IC50 are listed in Table 2. The fractional inhibitory concentration indexes (FICIs) of mixtures with various ratios of PEG-AgNPs and NaF lay between 1 and 1.3, showing no synergistic antibacterial effect from these two components. At high PEG-AgNPs to NaF ratios, the antibacterial effect mainly came from $\mathrm{Ag}$, whereas the color of the antibacterial effect mainly came from $\mathrm{F}$ at low ratios of PEG-AgNPs to NaF. When the ratio of PEG-AgNPs to NaF was decreased, less PEG-AgNPs and more $\mathrm{NaF}$ were needed to inhibit the growth of $S$. mutans. From the result above, NaF was not cytotoxic toward HGF-1 even at high concentrations. Therefore, by decreasing the ratio of PEG-AgNPs to NaF, the amount of PEG-AgNPs required to inhibit bacterial growth would be less, thus leading to less damage to HGF-1.

\section{Coloring Test}

The color characteristics for dentin specimens treated with various fluoridated PEG-AgNPs are shown in Table 3. These data indicate that solutions containing various ratios of PEG-AgNP to NaF did not introduce any adverse coloring effect on dentin blocks, whereas an SDF application caused distinct blackening (Figure 3). No significant differences in color parameters $\mathrm{L}^{*}, \mathrm{C}^{*}$ and $\mathrm{h}^{*}$ were found between dentin blocks treated with fluoridated PEGAgNPs and water (negative control) $(p>0.05)$. On the contrary, the $L^{*}, C^{*}$ and $h^{*}$ values of specimens treated with SDF were significantly less than those of the other

Table I Antibacterial (S. mutans) and Cytotoxic Effect (HGF-I) of PEG-AgNPs, $\mathrm{AgNO}_{3}, \mathrm{NaF}$, and SDF

\begin{tabular}{|l|l|l|l|l|l|}
\hline \multirow{2}{*}{ Testing Compounds } & \multicolumn{2}{l|}{ IC50 Against S. mutans } & \multicolumn{2}{l|}{ IC50 Against HGF-I cells } & \multirow{2}{*}{$\boldsymbol{p}$ value } \\
\cline { 2 - 6 } & Silver (ppm) & Fluoride (ppm) & Silver (ppm) & Fluoride (ppm) \\
\hline $\mathrm{PEG-AgNPs}$ & $21.16 \pm 1.08$ & - & $42.36 \pm 1.12$ & - & $<0.00 \mathrm{I}$ \\
$\mathrm{AgNO}_{3}$ & $7.13 \pm 2.16$ & - & $0.23 \pm 0.04$ & - & $<0.00 \mathrm{I}$ \\
$\mathrm{NaF}$ & - & $32.96 \pm 2.86$ & - & $\mathrm{N} / \mathrm{A}^{\mathrm{a}}$ & $\mathrm{N} / \mathrm{A}$ \\
$\mathrm{SDF}$ & $9.67 \pm 1.55$ & $1.71 \pm 0.27$ & $32.78 \pm 0.47$ & $5.79 \pm 0.08$ & $<0.00 \mathrm{I}$ \\
\hline
\end{tabular}

Note: ${ }^{a}$ IC50 against HGF-I cells was not reached in medium containing up to II3I ppm F.

Table 2 Antibacterial Effects of Formulations at Various Ratios of PEG-AgNPs and NaF Against S. mutans

\begin{tabular}{|l|l|l|l|l|}
\hline & Testing Compound Stocks & $\mathbf{A g}^{+}: \mathbf{F}^{-}$Ratio & IC50 Against S. mutans & \multirow{2}{*}{ FICI Index } \\
\cline { 3 - 4 } & & Silver (ppm) & Fluoride (ppm) & \\
\hline I2,800 ppm AgNPs + 25,000 ppm NaF & I: 0.88 & $14.01 \pm 3.06$ & $12.38 \pm 2.70$ & 1.04 \\
6400 ppm AgNPs + 25,000 ppm NaF & I: 1.77 & $12.86 \pm 0.69$ & $22.72 \pm 1.22$ & 1.30 \\
I 600 ppm AgNPs + 25,000 ppm NaF & I: 7.07 & $4.02 \pm 0.22$ & $28.40 \pm 1.55$ & 1.05 \\
400 ppm AgNPs + 25,000 ppm NaF & I: 28.28 & $1.23 \pm 0.07$ & $34.84 \pm 1.89$ & 1.12 \\
\hline
\end{tabular}


Table 3 Coloring Parameters of Tooth Slices Treated with Fluoridated PEG-AgNPs, SDF, and Water

\begin{tabular}{|l|l|l|l|l|l|l|l|l|}
\hline & A 38\% SDF & $\begin{array}{l}\text { B 12,800 ppm } \\
\text { AgNPs + 2.5\% NaF }\end{array}$ & $\begin{array}{l}\text { C 6400 ppm } \\
\text { AgNPs + 2.5\% } \\
\text { NaF }\end{array}$ & $\begin{array}{l}\text { D 1600 ppm } \\
\text { AgNPs + 2.5\% } \\
\text { NaF }\end{array}$ & $\begin{array}{l}\text { E } 400 \text { ppm } \\
\text { AgNPs + 2.5\% } \\
\text { NaF }\end{array}$ & F Water & p value & $\begin{array}{l}\text { Bonferroni } \\
\text { Test }\end{array}$ \\
\hline$\Delta \mathrm{L}^{*}$ & $16.2 \pm 0.8$ & $93.4 \pm 11.3$ & $88.7 \pm 9.8$ & $86.9 \pm 7.5$ & $97.9 \pm 3.7$ & $90.8 \pm 9.9$ & $<0.001$ & $\mathrm{~A}<\mathrm{B}, \mathrm{C}, \mathrm{D}, \mathrm{E}, \mathrm{F}$ \\
$\Delta \mathrm{C}^{*}$ & $7.6 \pm 0.6$ & $41.7 \pm 3.5$ & $41.5 \pm 7.1$ & $37.4 \pm 1.0$ & $44.4 \pm 7.7$ & $39.9 \pm 7.0$ & $<0.001$ & $\mathrm{~A}<\mathrm{B}, \mathrm{C}, \mathrm{D}, \mathrm{E}, \mathrm{F}$ \\
$\Delta \mathrm{h}^{*}$ & $66.4 \pm 1.4$ & $90.9 \pm 2.2$ & $90.2 \pm 1.7$ & $89.2 \pm 2.7$ & $91.0 \pm 2.5$ & $91.0 \pm 2.5$ & $<0.001$ & $\mathrm{~A}<\mathrm{B}, \mathrm{C}, \mathrm{D}, \mathrm{E}, \mathrm{F}$ \\
$\Delta \mathrm{a}^{*}$ & $3.0 \pm 0.3$ & $-0.7 \pm 1.6$ & $-0.2 \pm 1.4$ & $0.5 \pm 1.8$ & $-0.5 \pm 1.6$ & $-0.8 \pm 1.8$ & $\mathrm{~N} / \mathrm{A}$ & $\mathrm{N} / \mathrm{A}$ \\
$\Delta \mathrm{b}^{*}$ & $6.9 \pm 0.6$ & $41.7 \pm 3.5$ & $41.5 \pm 7.1$ & $37.3 \pm 0.9$ & $44.4 \pm 7.7$ & $39.8 \pm 6.9$ & $\mathrm{~N} / \mathrm{A}$ & $\mathrm{N} / \mathrm{A}$ \\
$\Delta \mathrm{E}^{*}$ & 81.6 & 3.2 & 2.8 & 4.6 & 8.5 & N/A & N/A & N/A \\
\hline
\end{tabular}

Notes: $\Delta \mathrm{L}^{*}$ was the difference of lightness axis ranged from black $(0)$ to white $(100) . \Delta \mathrm{a}^{*}$ was the difference of axis ranged from red $\left(+\mathrm{a}^{*}\right)$ to green $\left(-\mathrm{a}^{*}\right)$. $\Delta \mathrm{b}^{*}$ was the difference of axis ranged from yellow $\left(+b^{*}\right)$ to blue $\left(-b^{*}\right) . \Delta h^{*}$ means the difference of hue. $\Delta \mathrm{C}^{*}$ means the difference of chroma. $\Delta \mathrm{E}^{*}$ was the difference of color calculated based on the equation $\Delta \mathrm{E}^{*}=\left[\left(\Delta \mathrm{L}^{*}\right) 2+\left(\Delta \mathrm{a}^{*}\right) 2+\left(\Delta \mathrm{b}^{*}\right) 2\right]^{1 / 2}$.

Abbreviation: N/A, not applicable.

three groups $(p<0.001)$. Based on the color difference formula, the mean values of the $\mathrm{L}^{*}, \mathrm{a}^{*}$ and $\mathrm{b}^{*}$ color coordinates were used to calculate the color differences between treated dentin blocks and the negative control. Samples applied with SDF acquired a very high $\Delta \mathrm{E}^{*}$ value (81.6), whereas those treated with fluoridated PEGAgNPs yielded low $\Delta \mathrm{E}^{*}$ values (2.8 to 8.5 ). This means that the colors of samples treated with SDF were significantly different from those treated with water, whereas fluoridated PEG-AgNPs-treated samples gave a similar color as the negative control.

\section{Discussion}

This study aimed to develop a novel anti-caries agent that does not color teeth. For development of a potential agent to be used in the human oral cavity, it is important that the synthesis does not involve any toxic or harmful substances. In previous studies, the synthesis system commonly involved sodium borohydride as the reducing agent, which is toxic and causes serious burns or irritation if ingested or inhaled and if it comes in contact with the skin. ${ }^{15}$ This study used thiolated PEG as both a reducing agent and a capping agent for the formation of AgNPs. It has received safety approval from the FDA. The resulting product was washed thoroughly to remove any unreacted substance, so PEG-AgNPs in water was obtained without impurities. In addition, it was difficult to prepare small monodispersed AgNPs with a diameter of less than $10 \mathrm{~nm}$. The preparation always involves the excess use of a strong reducing agent, such as sodium borohydride, as previously mentioned. ${ }^{16}$ AgNPs synthesized in a higher temperature can be larger in size and more liable for further aggregation. ${ }^{17}$ The synthetic method used was simple and convenient, as neither the constant flow of inert gas nor heating was required. The low preparation temperature $\left(4{ }^{\circ} \mathrm{C}\right)$ used in this study allowed for the formation of stable small monodispersed AgNPs (ca. $3 \mathrm{~nm}$ ), which is difficult to achieve otherwise. The UV-vis spectrum of the

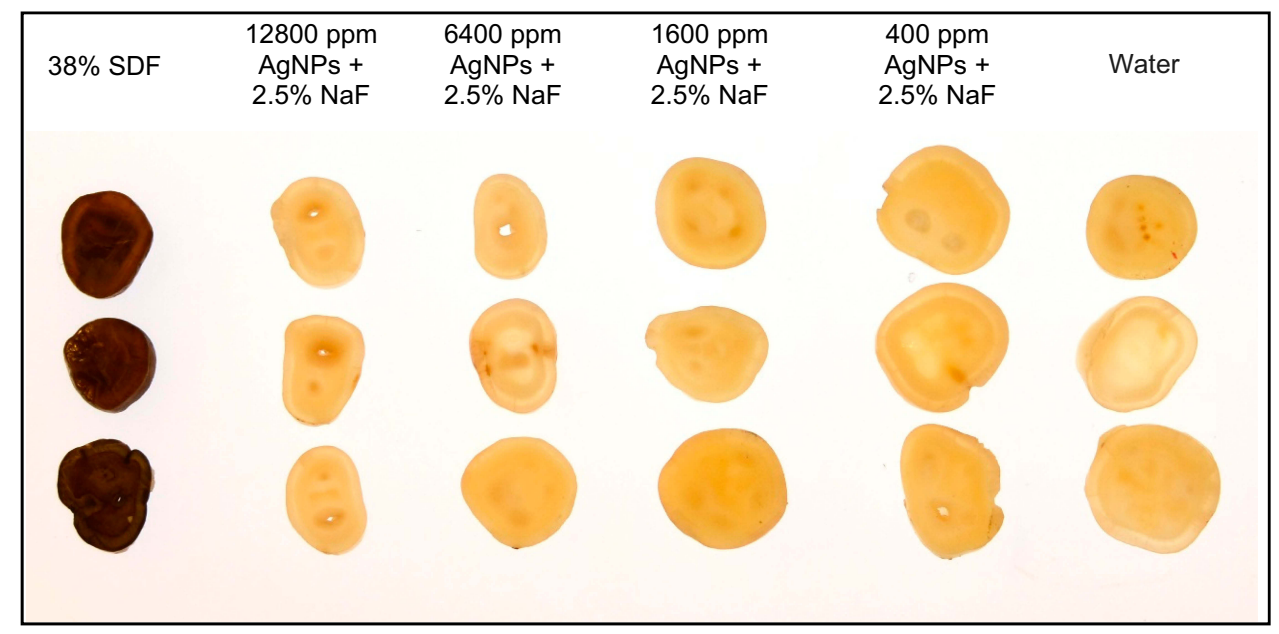

Figure 3 Staining effect on tooth slices treated with fluoridated PEG-AgNPs, NaF, SDF, and water. 
PEG-AgNPs did not show any characteristic SPR band. This is in agreement with a previous study showing that AgNPs smaller than $5 \mathrm{~nm}$ have a significantly broadened plasmon band that may cause them to lose their distinct spectral features. ${ }^{18}$

The mechanism of the antibacterial and cytotoxic effect of AgNPs is still under debate. Studies have shown that AgNPs itself was not toxic, whereas the toxicity was determined by the released $\mathrm{Ag}^{+}$ions. On the other hand, the contact of AgNPs with bacterial cells gave rise to a toxic effect, whereas the effect of released $\mathrm{Ag}^{+}$ions was not significant. Some other studies demonstrated the toxicity of both AgNPs and the released $\mathrm{Ag}^{+}$ions. ${ }^{19}$ The toxicity contribution of AgNPs and released $\mathrm{Ag}^{+}$ions depends on the size of the AgNPs. The toxicity of AgNPs with a size larger than $10 \mathrm{~nm}$ is solely due to the release of $\mathrm{Ag}^{+}$ions, whereas that of AgNPs smaller than $10 \mathrm{~nm}$ contributed to both the AgNPs and released $\mathrm{Ag}^{+}$ion effect. The toxicity of AgNPs smaller than $10 \mathrm{~nm}$ was dominated by the released $\mathrm{Ag}^{+}$ions, whereas the antibacterial effect of larger AgNPs contributed comparably to the released $\mathrm{Ag}^{+}$ion and AgNPs. ${ }^{20}$ It seemed that the antibacterial and cytotoxic effects of AgNPs prepared in this study were due to the AgNPs themselves rather than the released $\mathrm{Ag}^{+}$ion. Freshly prepared AgNPs were free of an $\mathrm{Ag}^{+}$ion because any unreacted $\mathrm{Ag}^{+}$ions were removed via the filtered centrifugal unit. Moreover, TEM micrographs showed that no significant loss of AgNPs' size occurred upon storage. This showed that the AgNPs were very stable and did not demonstrate a significant release of $\mathrm{Ag}^{+}$ions under the conditions in which the experiments were carried out.

Many studies were conducted to determine the toxicity of AgNPs toward various kinds of cells, including bacteria, yeast, algae, and mammalian cells in vitro, as well as non-mammalian models in vivo. Minimal inhibitory concentration (MIC) is a common method to measure the toxicity of materials. It is easy to perform, but it is difficult to receive accurate results because the MIC results were obtained in a 2-fold dilution series. The errors in a doubling dilution series represent at minimum a 2-fold difference. Also, cumulative deviation was obtained through manual preparation of a 2 -fold dilution series. ${ }^{21}$ The dose-response inhibition of bacterial growth can be modeled as a curve using spectrophotometry to yield IC50. The results of IC50 are expected to be more robust than the MIC value on the measurement of antibacterial effect. However, no consistent data exist due to the variations in studies. $^{22}$ The antibacterial and cytotoxicity effects of AgNPs are highly dependent on the coating agent, size, shape, monodispersity, purity, and cell culture conditions and media. Studies have found that smaller AgNPs possess a higher antibacterial effect. ${ }^{23}$ By decreasing the size of AgNPs, the actual silver content used can be reduced to provide the desired antibacterial effect. When AgNPs are used as anti-caries agent, only a minute amount (ca. 10 $\mu \mathrm{L}$, ng level of $\mathrm{Ag}$ ) is applied once or twice yearly with a micro-brush onto a decayed tooth surface with no living cells. Moreover, research has shown that mammalian cells are most resistant to AgNPs among the tested species. ${ }^{24}$ Therefore, a therapeutic window exists where mammalian cells are not harmed, whereas bacterial cells are killed. Our result is in agreement with these studies, showing that the antibacterial dose of our PEG-AgNP against $S$. mutans is lower than its cytotoxic concentration against HGF-1 cells, allowing for a range of concentrations where S. mutans is inhibited without harming HGF-1 cells. PEGAgNPs that do not stay on the tooth cavity will be removed from the body with a mouth rinse or will be swallowed at a much diluted concentration. This fluoridated PEG-AgNPs anti-caries agent should not exert any cytotoxic effect on patients.

For the purpose of investigating the potential use of this PEG-AgNP as an anti-caries agent, its compatibility with a fluoride source $(\mathrm{NaF})$ was tested. Fluorides can inhibit tooth demineralization and aid in remineralization during the early stage of caries, so they can prevent as well as arrest caries. ${ }^{2}$ The commercially available anti-caries agent, SDF, includes fluoride as one of its main ingredients. The PEG-AgNPs reported here were highly compatible with $\mathrm{NaF}$, and they did not show precipitation or aggregation. Unlike with SDF, where the stoichiometric ratio of silver and fluoride concentrations (1:1) was fixed, PEG-AgNPs can be mixed with $\mathrm{NaF}$ at various concentrations depending on the requirement of antibacterial $\mathrm{Ag}$ and remineralizing $\mathrm{F}$. The ratio and concentration of each component can be fine-tuned to balance the antibacterial and cytotoxic effect, as well as the remineralization power and risk of fluorosis development. For the purpose of testing these possibilities, various amounts of PEG-AgNP were mixed with a fixed amount of $2.5 \% \mathrm{NaF}(11,310 \mathrm{ppm}$ F). The concentration of PEG-AgNP ranged from 12,800 to $400 \mathrm{ppm} \mathrm{Ag}$. The FICIs of PEG-AgNP and NaF mixtures of various ratios were found to be between 1 and 1.3, meaning that no synergistic antibacterial effect existed upon mixing the two compounds. This is in agreement 
with a previous study showing that the addition of fluoride does not influence the antibacterial effect of silver against S. mutans. $^{25}$ However, $\mathrm{NaF}$ exhibited IC50 against S. mutans at about $33 \mathrm{ppm} \mathrm{F}$ and showed no cytotoxic effect toward HGF-1 cells up to 1131 ppm F. By combining PEG-AgNP and NaF, the mixture can achieve a similar antibacterial effect while having a much lower cytotoxicity. The combination of PEG-AgNP and NaF has broadened the therapeutic window where caries bacteria can be inhibited without harming human cells.

The aesthetic outcome of dental treatment will be a main concern for patients. Instrumental measurements were used to quantify the color characteristics more precisely with a high reproducibility rather than assessing color differences with the naked eye, which could be nonobjective. Tooth discoloration resulting from the topical application of SDF is well known, but our product's aim is to be superior to SDF by not exerting a staining effect. The measurement of the color difference between the treatment and negative control in this study indicated that SDF caused obvious staining, whereas no significant staining by mixtures of PEG-AgNP and NaF was observed. The color difference value $\left(\Delta \mathrm{E}^{*}\right)$ resulting from solutions containing $\mathrm{PEG}-\mathrm{AgNP}$ and $\mathrm{NaF}$ ranged from 2.8 to 8.5 and could not be detected visually, whereas the discoloration due to $\operatorname{SDF}\left(\Delta \mathrm{E}^{*}=81.6\right)$ would not be accepted clinically because it was more than 10 times higher than the upper acceptable threshold. ${ }^{26}$

\section{Conclusion}

This study involved the preparation of water-soluble and biocompatible AgNPs via a green synthetic method without the use of a reducing agent. The PEG-AgNPs are stable at a $2.5 \% \mathrm{NaF}$ solution. Because it has antibacterial activity against $S$. mutans and no tooth-staining effect, fluoridated PEG-AgNPs can be used as an anti-caries agent.

\section{Ethics Approval}

This study received approval from the Institutional Review Board at the University of Hong Kong under process number IRB UW14-529. Extracted sound human third molars were collected with the patients' consent provided in hardcopy forms.

\section{Acknowledgment}

This study was supported by the National Natural Science Foundation of China (NSFC) - General Program (2018)
81870812 and the General Research Fund (GRF) 17100218 of the Research Grant Council, Hong Kong.

\section{Disclosure}

The authors declare that they have no conflict of interest. This study has won the International Association for Dental Research (IADR) KULZER Travel Award, and part of this study will be presented in IADR General Session 2020 in March 2020 in Washington, DC, USA.

\section{References}

1. Chernousova S, Epple M. Silver as antibacterial agent: ion, nanoparticle, and metal. Angew Chem Int Ed. 2013;52(6):1636-1653. doi:10.1002/anie.201205923

2. Mei ML, Lo ECM, Chu CH. Arresting dentine caries with silver Diamine fluoride: what's behind it? J Dent Res. 2018;97(7):751-758. doi: $10.1177 / 0022034518774783$

3. Gao SS, Zhang S, Mei ML, Lo EC, Chu CH. Caries remineralisation and arresting effect in children by professionally applied fluoride treatment - a systematic review. BMC Oral Health. 2016;16:12. doi:10.1186/s12903-016-0171-6

4. Yu OY, Mei ML, Zhao IS, Li QL, Lo EC, Chu CH. Remineralisation of enamel with silver diamine fluoride and sodium fluoride. Dent Mater. 2018;34(12):e344-e352. doi:10.1016/j.dental.2018.10.007

5. Tejamaya M, Romer I, Merrifield RC, Lead JR. Stability of citrate, PVP, and PEG coated silver nanoparticles in ecotoxicology media. Environ Sci Technol. 2012;46(13):7011-7017. doi:10.1021/ es2038596

6. Reidy B, Haase A, Luch A, Dawson K, Lynch I. Mechanisms of silver nanoparticle release, transformation and toxicity: a critical review of current knowledge and recommendations for future studies and applications. Materials. 2013;6(6):2295. doi:10.3390/ma6062295

7. Nowack B, Krug H, Height M. 120 years of nanosilver history: implications for policy makers (vol 45, pg 1177, 2011). Environ Sci Technol. 2011;45(7):3189. doi:10.1021/es200435m

8. Yin IX, Yu OY, Zhao IS, Mei ML, Li QL, Tang J, Chu CH. Developing biocompatible silver nanoparticles using epigallocatechin gallate for dental use. Arch Oral Biol. 2019;102:106-112. doi:10.1016/j.archoralbio.2019.03.022

9. Dias HB, Bernardi MIB, Marangoni VS, de Abreu Bernardi AC, de Souza Rastelli AN, Hernandes AC. Synthesis, characterization and application of $\mathrm{Ag}$ doped $\mathrm{ZnO}$ nanoparticles in a composite resin. Mater Sci Eng C Mater Biol Appl. 2019;96:391-401. doi:10.1016/j. msec.2018.10.063

10. Teixeira JA, AVCe S, Santos V Jr, et al. Effects of a new nano-silver fluoride-containing dentifrice on demineralization of enamel and adhesion and acidogenicity. Int J Dent. 2018;2018. doi:10.1155/ 2018/1351925

11. Sawhney AS, Drumheller PD. Chapter II.4 - polymer synthesis. In: Mikos CWPG, Langer LVMS, editors. Frontiers in Tissue Engineering. Oxford: Pergamon; 1998:83-106.

12. Ivask A, Kurvet I, Kasemets K, et al. Size-dependent toxicity of silver nanoparticles to bacteria, yeast, algae, Crustaceans and Mammalian cells in vitro. PLoS One. 2014;9:7. doi:10.1371/journal. pone. 0102108

13. Gold OG, Jordan HV, Van Houte J. A selective medium for Streptococcus mutans. Arch Oral Biol. 1973;18(11):1357-1364. doi:10.1016/0003-9969(73)90109-X

14. Besinis A, De Peralta T, Handy RD. Inhibition of biofilm formation and antibacterial properties of a silver nano-coating on human dentine. Nanotoxicology. 2014;8(7):745-754. doi:10.3109/ 17435390.2013 .825343 
15. Dos Santos VE, Filho AV, Ribeiro Targino AG, et al. A new "SilverBullet" to treat caries in children - nano silver fluoride: a randomised clinical trial. $J$ Dent. 2014;42(8):945-951. doi:10.1016/j. jdent.2014.05.017

16. Agnihotri S, Mukherji S, Mukherji S. Size-controlled silver nanoparticles synthesized over the range 5-100 nm using the same protocol and their antibacterial efficacy. RSC $A d v$. 2014;4(8):3974-3983 doi:10.1039/C3RA44507K

17. Liu M, Gao X, Pan F, et al. Effect of pyrene on formation of natural silver nanoparticles via reduction of silver ions by humic acid under UV irradiation. Chemosphere. 2020;247:125937. doi:10.1016/j. chemosphere.2020.125937

18. Amendola V, Bakr OM, Stellacci F. A study of the surface plasmon resonance of silver nanoparticles by the discrete dipole approximation method: effect of shape, size, structure, and assembly. Plasmonics. 2010;5(1):85-97. doi:10.1007/s11468-009-9120-4

19. Marin S, Vlasceanu GM, Tiplea RE, et al. Applications and toxicity of silver nanoparticles: a recent review. Curr Top Med Chem. 2015;15(16):1596-1604. doi:10.2174/1568026615666150414142209

20. Cho YM, Mizuta Y, Akagi JI, Toyoda T, Sone M, Ogawa K. Sizedependent acute toxicity of silver nanoparticles in mice. $J$ Toxicol Pathol. 2018;31(1):73-80. doi:10.1293/tox.2017-0043
21. Brennan-Krohn T, Smith KP, Kirby JE. The poisoned well: enhancing the predictive value of antimicrobial susceptibility testing in the era of multidrug resistance. J Clin Microbiol. 2017;55(8):2304-2308. doi:10.1128/JCM.00511-17

22. Liao C, Li Y, Tjong SC. Bactericidal and cytotoxic properties of silver nanoparticles. Int J Mol Sci. 2019;20:2. doi:10.3390/ ijms20020449

23. Kumari M, Pandey S, Giri VP, et al. Tailoring shape and size of biogenic silver nanoparticles to enhance antimicrobial efficacy against MDR bacteria. Microb Pathog. 2017;105:346-355. doi:10.1016/j.micpath.2016.11.012

24. Jiravova J, Tomankova KB, Harvanova M, et al. The effect of silver nanoparticles and silver ions on mammalian and plant cells in vitro. Food Chem Toxicol. 2016;96:50-61. doi:10.1016/j.fct.2016.07.015

25. Yu OY, Zhao IS, Mei ML, Lo EC, Chu CH. Effect of silver nitrate and sodium fluoride with tri-calcium phosphate on streptococcus mutans and demineralised dentine. Int J Mol Sci. 2018;19(5). doi:10.3390/ijms19051288

26. Westland S, Luo W, Li Y, Pan Q, Joiner A. Investigation of the perceptual thresholds of tooth whiteness. J Dent. 2017;67s:S11S14. doi:10.1016/j.jdent.2017.09.013
International Journal of Nanomedicine

\section{Publish your work in this journal}

The International Journal of Nanomedicine is an international, peerreviewed journal focusing on the application of nanotechnology in diagnostics, therapeutics, and drug delivery systems throughout the biomedical field. This journal is indexed on PubMed Central, MedLine, CAS, SciSearch ${ }^{\circledR}$, Current Contents ${ }^{\circledR} /$ Clinical Medicine,

\section{Dovepress}

Journal Citation Reports/Science Edition, EMBase, Scopus and the Elsevier Bibliographic databases. The manuscript management system is completely online and includes a very quick and fair peer-review system, which is all easy to use. Visit http://www.dovepress.com/ testimonials.php to read real quotes from published authors. 